\title{
NSAR nach ERCP für (fast) jeden Patienten!
}

Bisher herrschte keine gute Evidenz, ob Antiphlogistika das Risiko einer Post-ERCP-Pankreatitis reduzieren können. Eine aktuelle Metaanalyse kommt jetzt zu einem eindeutigen Schluss.

Hintergrund und Fragestellung: Die Post-ERCP-Pankreatitis (PEP) ist eine gefürchtete Komplikation in der endoskopischen Diagnostik und Therapie. Verschiedene Strategien zur Risikoreduktion werden diskutiert und angewendet, wobei auch die medikamentöse Prophylaxe zum Einsatz kommt. Diclofenac und Indomethacin sind die meist untersuchten Substanzen zur Prophylaxe der PEP. Dennoch gibt es keine prospektiven, multizentrischen Studien mit ausreichender Patientenzahl zur validen Beurteilung der Effizienz. Das Ziel der hier besprochenen Metaanalyse war es, alle prospektiven Volltextstudien zur

Patai Á et al. Indomethacin and diclofenac in the prevention of post-ERCP pancreatitis Gastrointest Endosc 2017; 85: 1144-56 Effizienz von Diclofenac oder Indomethacin im Vergleich mit Placebo oder Nichtbehandlung bezüglich der PEPProphylaxe zu untersuchen.
Patienten und Methoden: Diese Metaanalyse umfasst 4.741 Patienten aus 17 Studien, die in einer systematischen Datenbankanalyse (PubMed, Scopus, Web of Science und Cochrane) bis zum 30. Juni 2016 ausgewählt worden waren.

Ergebnisse: Diclofenac oder Indomethacin zeigten ein signifikant reduziertes relatives Risiko einer PEP auf 0,6 (95\%-Konfidenzintervall $[\mathrm{KI}], 0,46-0,78 ; \mathrm{p}=0,0001)$. Die number needed to treat (NNT) betrug 20, Unterschiede in der Effektivität der PEP-Prophylaxe fanden sich nicht $(\mathrm{p}=0,98)$. Der Zeitpunkt der Gabe hatte keine Bedeutung für die Wirksamkeit, wie auch das Risiko für eine PEP keine Vorhersage für den Therapieerfolg zuließ $(p=0,99$ respektive $p=0,6923)$. Die rektale Applikation zeigte einen Trend zur besseren Wirksamkeit $(p=0,1507$, NNT 19). Patienten mit Nierenversagen wurden nicht behandelt.

Schlussfolgerungen: Indomethacin oder Diclofenac, bevor oder kurz nach einer ERCP in rektaler Applikationsform, sind kostengünstig, sicher und effektiv. Die Prophylaxe wird für alle $\mathrm{Pa}$ tienten (ohne Nierenversagen) empfohlen.

\section{- Kommentar von Holger H. Lutz, Radebeul}

\section{Gutes Nutzen-/Risiko-Verhältnis für NSAR in der Pankreatitisprophylaxe}

Bereits in den 1970er-Jahren wurde Indomethacin im Tierexperiment zur Therapie der Pankreatitis eingesetzt [1]. In kleineren Studien wird seit zirka zehn Jahren immer wieder der prophylaktische Effekt untersucht - mit unterschiedlichen Ergebnissen, die selbst in Metaanalysen nicht immer kongruent waren $[2,3]$. Auffällig ist die aus unserer Sicht hohe Rate an Post-ERCPPankreatitiden in den meisten der eingeschlossenen Studien (in den Kontrollgruppen teils weit > 10\%). Die Gesamtmortalität innerhalb von 30 Tagen nach ERC beträgt 5,3\%, die ERCPspezifische Mortalität 0,4-0,5\%. Die Komplikationsrate sollte heutzutage mit modernen Endoskopen in den Händen erfahrener Untersucher mit den entsprechenden Kanülierungstechniken deutlich niedriger liegen (PEP-Rate $<5 \%$ ).

Die vorgestellte Metaanalyse umfasst mit fast 5.000 Patienten eine große, aber heterogene Gruppe, was die Bewertung der Studie nicht einfach macht. Dennoch ist das Ergebnis überzeugend: Mit einer NNT, die nur 1/10 der NNT moderner Antikoagulanzien beträgt, und dabei ein deutlich niedriges Risikoprofil hat, ist der NSAR-Einsatz in der standardisierten PEP-Prophylaxe angekommen. Aufgrund des niedrigen Risikos (kein Nachweis unerwünschter Ereignisse in den eingeschlossenen Studien) ist ein Verzicht auf die PEP-Prophylaxe mit Indomethacin und Diclofenac obsolet. Nicht zuletzt aufgrund der uneinheitlichen Datenlage ist eine Risikoselektionierung für die Notwendigkeit der Prophylaxe aus unserer Sicht ebenfalls nicht sinnvoll.

Mit dem konsequenten Einsatz der PEP-Prophylaxe - und hochqualitativer ERCP-Technik - kann das Risiko einer PEP signifikant reduziert werden; mit geringem Aufwand und nied- rigem Risiko. Fazit: Auch wenn die Datenlage nicht einheitlich ist, sollte jeder Patient (ohne Nierenversagen oder anderweitige Kontraindikation), der einer ERCP zugeführt wird, eine PEP-Prophylaxe mit Indomethacin oder Diclofenac erhalten.
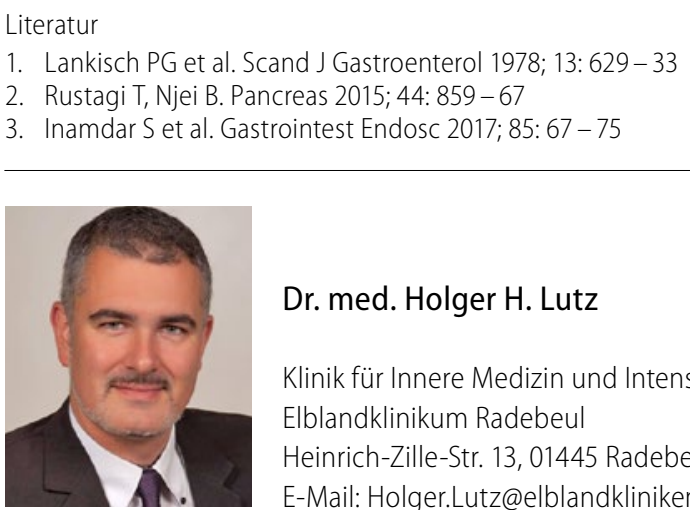

Dr. med. Holger H. Lutz

Klinik für Innere Medizin und Intensivmedizin

Elblandklinikum Radebeul

Heinrich-Zille-Str. 13, 01445 Radebeul

E-Mail: Holger.Lutz@elblandkliniken.de

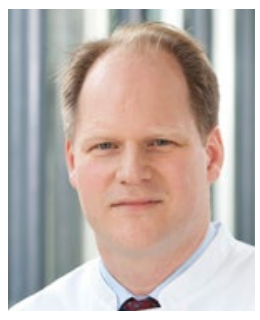

Prof. Dr. med. Jens J.W. Tischendorf

Klinik für Innere Medizin und

Gastroenterologie, Rhein-Maas Klinikum der Städteregion Aachen/Würselen

E-Mail: Jens.Tischendorf@rheinmaasklinikum.de 